\title{
From one- to two-dimensional solitons in the Ginzburg-Landau model of lasers with frequency-selective feedback
}

\author{
P. V. Paulau, ${ }^{1,2}$ D. Gomila, ${ }^{1}$ P. Colet,${ }^{1}$ B. A. Malomed,${ }^{3}$ and W. J. Firth ${ }^{4}$ \\ ${ }^{1}$ IFISC, Instituto de Física Interdisciplinar y Sistemas Complejos (CSIC-UIB), Campus Universitat Illes Balears, \\ E-07071 Palma de Mallorca, Spain \\ ${ }^{2}$ Institute of Physics, Nezavisimosty av. 68, 220072 Minsk, Belarus \\ ${ }^{3}$ Department of Physical Electronics, School of Electrical Engineering, Faculty of Engineering, Tel Aviv University, 69978 Tel Aviv, Israel \\ ${ }^{4}$ Department of Physics, University of Strathclyde, 107 Rottenrow East, Glasgow G4 0NG, United Kingdom
}

(Received 7 February 2011; published 23 September 2011)

\begin{abstract}
We use the cubic complex Ginzburg-Landau equation linearly coupled to a dissipative linear equation as a model for lasers with an external frequency-selective feedback. This system may also serve as a general pattern-formation model in media driven by an intrinsic gain and selective feedback. While, strictly speaking, the approximation of the laser nonlinearity by a cubic term is only valid for small field intensities, it qualitatively reproduces results for dissipative solitons obtained in models with a more complex nonlinearity in the whole parameter region where the solitons exist. The analysis is focused on two-dimensional stripe-shaped and vortex solitons. An analytical expression for the stripe solitons is obtained from the known one-dimensional soliton solution, and its relation with vortex solitons is highlighted. The radius of the vortices increases linearly with their topological charge $m$, therefore the stripe-shaped soliton may be interpreted as the vortex with $m=\infty$, and, conversely, vortex solitons can be realized as unstable stripes bent into stable rings. The results for the vortices are applicable for a broad class of physical systems.
\end{abstract}

DOI: 10.1103/PhysRevE.84.036213

PACS number(s): $89.75 . \mathrm{Kd}, 42.65 . \mathrm{Tg}, 42.81 . \mathrm{Dp}$

\section{INTRODUCTION}

The field of spatial pattern formation in nonlinear dissipative media has grown significantly in the past decades (see reviews [1-11]). The research in this field was strongly driven by the interest in self-localized states ("dissipative solitons"), which arise in sundry systems, including various optical settings. The studies of the transverse dissipativepattern formation in nonlinear optics have been focused on systems such as lasers with saturable gain and absorption (see Chap. 5 in Refs. [7] and [12]), nonlinear cavities pumped by a holding beam [13], and coupled cavities [14].

More recently, broad-area vertical-cavity surface-emitting lasers (VCSELs), coupled to an external frequency-selective feedback (FSF) element, have been considered in the context of the pattern formation [15-20]. This system may be modeled by a complex Ginzburg-Landau equation (CGLE) coupled to a linear one, which represents, respectively, the nonlinear and linear subsystems. In this setting, the (complex) intra-VCSEL field features nonlinear spatiotemporal dynamics due to the two-way coupling between the optical field and the inversion of the electronic population (driven by the injection current), while the separate feedback field obeys a linear equation [21] which is linearly coupled to the main equation for the intraVCSEL field. Previous studies have modeled the VCSELFSF system using various approximations, which display a rich variety of spatiotemporal regimes, including solitons with complex dynamics $[15,16]$, side-mode solitons supported by the external cavity [16], and solitary vortices [17].

Although the use of the cubic nonlinearity for modeling the VCSEL is a simplification which is only strictly valid for lowfield intensities, the cubic CGLE enables analytical treatment of the problem in some cases, and also provides a direct insight into the underlying physical mechanisms. In this paper, we demonstrate that the cubic nonlinearity produces qualitatively the same results, concerning the formation and dynamics of solitons, as more complex (saturable) nonlinearities, thus justifying the use of the system of the CGLE coupled to the linear equation as a basic model of the VCSEL-FSF system.

The paper is organized as follows. In Sec. II we present the model and its basic behavior. In Sec. III we revisit the analytical one-dimensional (1D) solution of our CGLE-FSF model and produce its analytically found bifurcation diagram. In Sec. IV, which is the core part of the paper, we report our findings for two-dimensional (2D) solutions. The paper is concluded by Sec. V.

\section{THE SYSTEM AND MODEL}

Following Ref. [17], we start from the general model describing the VCSEL coupled to the frequency-selective feedback without delay:

$$
\left\{\begin{aligned}
\frac{\partial E}{\partial t}= & -\kappa(1+i \alpha) E+\kappa(1+i \alpha) \mu \frac{E}{1+|E|^{2}} \\
& -i \Delta_{\perp} E+F-i \omega_{m} E, \\
\frac{\partial F}{\partial t}= & -\lambda F+\sigma \lambda E
\end{aligned}\right.
$$

where $\kappa$ is the cavity decay rate, $\alpha$ is the phase-amplitude coupling factor, $\mu$ is the pump current, normalized to be 1 at the threshold in the absence of the external feedback, and $\Delta_{\perp}$ is the transverse Laplacian accounting for the diffraction in the paraxial approximation. Relating to the feedback, $\lambda$ stands for the width of the frequency filter, and $\sigma$ its strength in units of $\kappa$, i.e., the minimum threshold is reduced from $\mu=1$ at $\sigma=0$ to $\mu=1-\sigma / \kappa$. Finally, because we choose our reference frequency to coincide with the maximum of the frequency-selective feedback profile, $\omega_{m}$ is the detuning, with respect to the reference frequency, of the solitary laser at threshold. 
Truncating the Taylor expansion of the saturable nonlinearity at the third order, Eq. (1) becomes a special case of a general system consisting of a cubic CGLE coupled to a linear equation:

$$
\left\{\begin{array}{l}
\frac{\partial E}{\partial t}=g_{0} E+g_{2}|E|^{2} E+(d+i D) \Delta_{\perp} E+F, \\
\frac{\partial F}{\partial t}=-\lambda F+\tilde{\sigma} E,
\end{array}\right.
$$

where $\operatorname{Re}\left(g_{0}\right)$ is the total linear loss (if negative) or gain (if positive), and $\operatorname{Im}\left(g_{0}\right)$ is the effective detuning between the laser and the filter. Further, $\operatorname{Re}\left(g_{2}\right)$ is the nonlinear loss (if negative) or gain (if positive), while $\operatorname{Im}\left(g_{2}\right)$ represents the strength of the self-focusing or defocusing nonlinearity. In this general setting, real parameters $D$ and $d$ account for transverse diffraction and diffusion of the field. These parameters become, for our particular case when Eq. (1) represents a laser with FSF,

$$
\begin{aligned}
g_{0} & =\kappa(1+i \alpha)(\mu-1)-i \omega_{m}, \\
g_{2} & =-\kappa(1+i \alpha) \mu, \\
\tilde{\sigma} & =\sigma \lambda,
\end{aligned}
$$

and $d=0$, the latter (absence of the diffusion of light) being relevant to optics in the spatial domain [22-25], but $d \neq 0$ occurs in other physical situations - in particular, in the temporal domain $[8,26]$.

Obviously, the stability of the nonlasing (zero) solutions of Eqs. (1) and (2) is identical. An interesting property exploited in previous papers is the existence, for positive $\alpha$ and for a range of negative values of $\omega_{m}$, of a stability region for the zero solution above the off-axis emission threshold (see Fig. 1). In this region, stable self-localized modes can be found $[15,16]$.

Strictly speaking, the truncation of the series mentioned above is valid only for $|E| \ll 1$. We observe, however, that the spatiotemporal patterns in model (2) are qualitatively the same as those previously discussed in more complicated models [15]. This provides a justification for the use of the CGLE coupled to FSF for modeling lasers with FSF even for high pump currents (hence for high-field values). For a set of parameters close to those adopted in Fig. 1, which are relevant to the laser dynamics, the following scenario is observed. For the pump current in the unstable region ( $\mu \approx 0.45$ ), small random perturbations of the zero solution grow exponentially, generating complex 2D spatiotemporal patterns, e.g., as shown in Fig. 2(a). If, starting from this

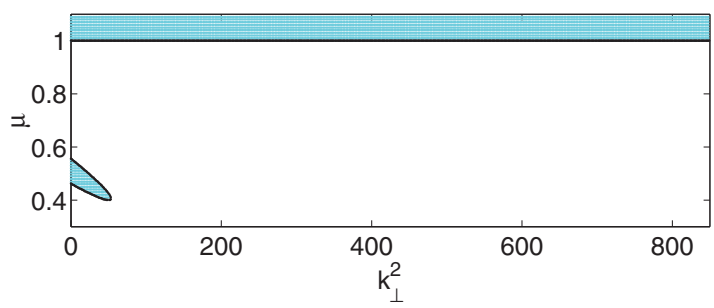

FIG. 1. (Color online) The marginal stability curve of the zero solution. The regions where the zero solution is unstable against perturbations with transverse wave number $k_{\perp}$ are shaded. The black line is the marginal-stability boundary. Throughout this paper we fix the following values of the parameters: $\omega_{m}=-250, \sigma=60$, $\kappa=100, \lambda=2.71, \alpha=5, d=0$, and $D=-1$. (a)

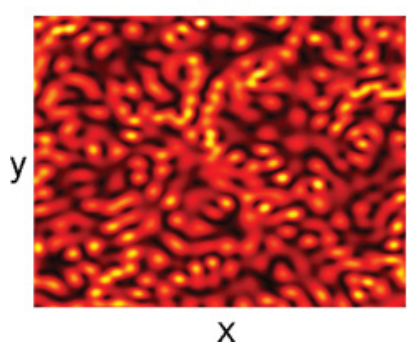

(b)

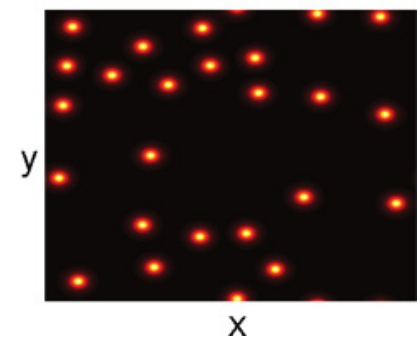

FIG. 2. (Color online) An instantaneous 2D amplitude profile corresponding to (a) a pump current at which the zero solution is unstable and a complex spatiotemporal regime arises, and (b) after having increased the pump current to a value for which the zero solution is stable, and the corresponding spatiotemporal pattern evolves into a set of fundamental solitons [27].

regime, the pump current $\mu$ is further increased up to a value at which the zero background is stable $(\mu=0.52)$, a transition is observed from the patterns built of densely packed filaments to a 2D set of isolated quiescent solitons [see Fig. 2(b)]. Initially, the distance between the solitons is small, and the interaction among them leads to annihilation events. However, when the soliton density becomes small enough, the resulting set of quiescent cavity solitons is quasistationary, featuring very weak interactions. A similar scenario is observed in one dimension, which is of special interest because exact analytical 1D self-localized solutions are available in the CGLE coupled to a linear equation [26], as we discuss in detail below.

Besides the fact that the model with the cubic nonlinearity provides qualitatively the same results as the one with the full saturation, the system including the cubic nonlinearity is relevant as a basic model for the study of generic features of the pattern formation in the class of dissipative nonlinear media to which the present laser model belongs [8].

\section{ONE-DIMENSIONAL SOLITONS}

In the case of one transverse dimension $\left(\Delta_{\perp}=\partial^{2} / \partial x^{2}\right)$ an exact analytical solution to Eqs. (2) can be found in the form $[8,26]$

$$
\left\{\begin{array}{l}
E=E_{\max }[\cosh (K x)]^{-1-i \beta} e^{i \omega t} \\
F=F_{\max }[\cosh (K x)]^{-1-i \beta} e^{i \omega t}
\end{array}\right.
$$

Substituting expressions (4) into Eqs. (2), we eliminate one amplitude,

$$
F_{\max }=\frac{\tilde{\sigma}}{\lambda+i \omega} E_{\max },
$$

and arrive at a quadratic equation for chirp $\beta$,

$$
\beta^{2}+3 \beta \frac{\operatorname{Re}\left[g_{2}(d-i D)\right]}{\operatorname{Im}\left[g_{2}(d-i D)\right]}-2=0,
$$

which yields a single physical root, due to the condition that the field intensity

$$
\left|E_{\max }\right|^{2}=3 \beta K^{2} \frac{d^{2}+D^{2}}{\operatorname{Im}\left[g_{2}(d-i D)\right]}
$$

must be positive. Note that $\beta$ does not depend on linear coefficient $g_{0}$, but only on the nonlinear one $g_{2}$, and on the parameters of the spatial coupling $d$ and $D$. Once $\beta$ is known, 
a complex algebraic equation involving $\omega$ and $K$ is obtained. By separating the real and imaginary parts of this equation, we obtain

$$
\begin{aligned}
K^{2} & =-\frac{\operatorname{Re}\left(g_{0}\right)}{\operatorname{Re}(\tilde{\beta})}-\frac{\tilde{\sigma} \lambda}{\lambda^{2}+\omega^{2}} \frac{1}{\operatorname{Re}(\tilde{\beta})}, \\
\tilde{\beta} & \equiv(1+i \beta)^{2}(d+i D) .
\end{aligned}
$$

Next, we obtain a cubic equation for $\omega$ :

$$
a_{3} \omega^{3}+a_{2} \omega^{2}+a_{1} \omega+a_{0}=0,
$$

where coefficients $a_{0}, a_{1}, a_{2}$, and $a_{3}$ depend on the system's parameters and on $\tilde{\beta}$ :

$$
\begin{aligned}
& a_{3}=\operatorname{Re}(\tilde{\beta}), \\
& a_{2}=\operatorname{Re}\left(g_{0}\right) \operatorname{Im}(\tilde{\beta})-\operatorname{Im}\left(g_{0}\right) \operatorname{Re}(\tilde{\beta}), \\
& a_{1}=\left(\tilde{\sigma}+\lambda^{2}\right) \operatorname{Re}(\tilde{\beta}), \\
& a_{0}=a_{2} \lambda^{2}+\tilde{\sigma} \lambda \operatorname{Im}(\tilde{\beta}) .
\end{aligned}
$$

In principle, Eq. (10) can be solved analytically, but it is more practically relevant to solve it numerically.

To summarize, the analytical solutions can be constructed according to the following scheme: (i) Solve Eq. (6) for $\beta$, and choose the proper root to satisfy the positivity of $\left|E_{\max }\right|^{2}$ as per Eq. (7). (ii) Solve Eq. (10) for $\omega$ with the coefficients defined by Eqs. (9) and (11), and $\beta$ produced by the previous step. (iii) Calculate $K$ using Eqs. (8) and (9), and $\omega$, with $\beta$ produced by two previous steps. (iv) Calculate $E_{\max }$ using Eq. (7). (v) Calculate $F_{\max }$ using Eq. (5).

Once all parameters of solution (4) are determined, the stability of this solution can be analyzed following, e.g., the numerical procedure developed in Ref. [17].

Figure 3(a) shows the bifurcation diagram for quiescent solitons (4), using parameter values typical to the lasers models. Point A corresponds to the pump threshold for the on-axis emission. For the pump levels $\mu$ between points $A$ and $\mathrm{B}$, the zero background is unstable as shown in Fig. 1. These points coincide with the origin of two branches of localized modes. The two branches collide at point $\mathrm{C}$ and disappear through a saddle-node bifurcation.

(a)

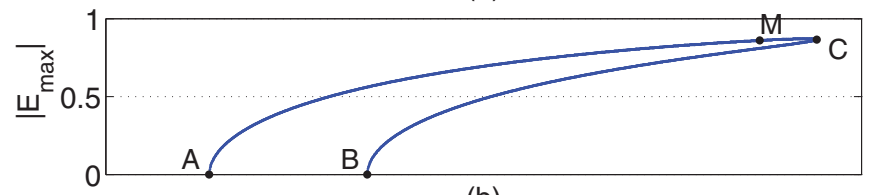

(b)

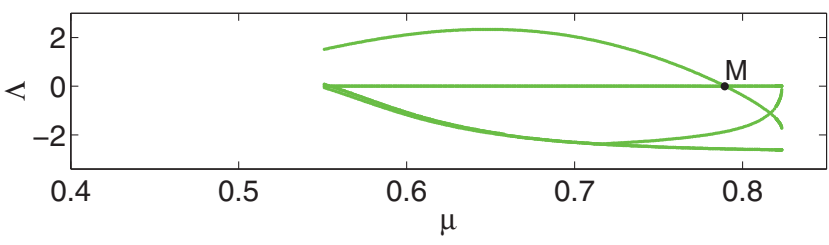

FIG. 3. (Color online) The bifurcation diagram for analytical solution (4). (a) The amplitude (absolute value of the field at the center of the fundamental soliton) as a function of the pump current. (b) The growth rate of unstable, neutral, and least-damped stable perturbation eigenmodes versus pump current $\mu$. Point $M$ designates the drift instability of the soliton.

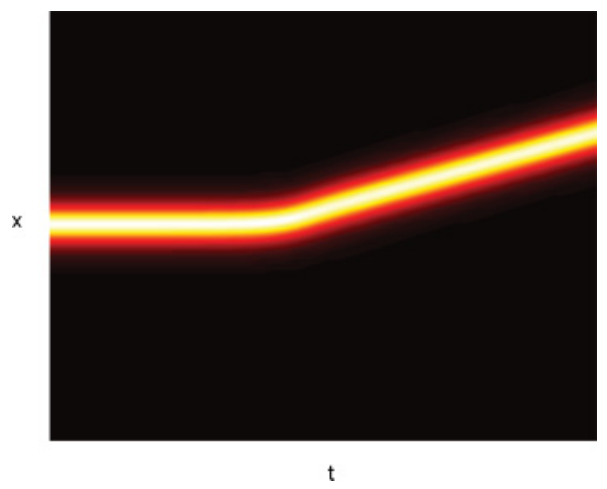

FIG. 4. (Color online) Spatiotemporal dynamics of the 1D soliton in the course of the development of the drifting instability, for $\mu=$ 0.7 .

Figure 3(b) shows the real part of the most relevant eigenvalues resulting from the stability analysis of the upper branch [the one connecting points C, M, and A in Fig. 3(a)]. The soliton solution is stable between points $\mathrm{C}$ and $\mathrm{M}$, and a drift instability appears at point $\mathrm{M}$ [16]. The instability spectrum is not shown between points $\mathrm{A}$ and $\mathrm{B}$ because the zero background solution is unstable in that region. The lower branch of the soliton solution, which connects points $\mathrm{B}$ and $\mathrm{C}$ in Fig. 3(a), is entirely unstable, as usual [28,29].

Direct simulations starting from the 1D analytical soliton in the unstable region (on the left-hand side of point $M$ in Fig. 3) show the development of the drift instability (see Fig. 4). Once the drift instability sets in, the soliton moves away from its original location at a constant speed.

\section{TWO-DIMENSIONAL LOCALIZED MODES : STRIPES, FUNDAMENTAL, AND VORTEX SOLITONS}

In two dimensions $\left(\Delta_{\perp}=\frac{\partial^{2}}{\partial x^{2}}+\frac{\partial^{2}}{\partial y^{2}}\right)$, 1D solution (4) can be generalized into a continuous family of stripe solitons, parametrized by the transverse wave number $k_{y}$ :

$$
\left\{\begin{array}{l}
E=E_{\max }[\cosh (K x)]^{-1-i \beta} e^{i \omega t} e^{i k_{y} y}, \\
F=F_{\max }[\cosh (K x)]^{-1-i \beta} e^{i \omega t} e^{i k_{y} y},
\end{array}\right.
$$

the difference from the 1D solution amounting to a modification of coefficient $g_{0}$, which is replaced by $\tilde{g}_{0}=g_{0}-(d+$ $i D) k_{y}^{2}$. An example of this solution is shown in Figs. 5(a) and 5(b). The whole family of the stripe solitons is represented, as a function of $k_{y}$, by plots displayed in Fig. 6. They exist only for values of $k_{y}^{2}$ below a certain value, beyond which the frequency shift introduced by $k_{y}$ pushes the solution outside the frequency range of the feedback filter. The solution with the largest amplitude, marked by a filled circle, occurs at $k_{y}=k_{y}^{c}$, corresponding to $\omega=0$, where the feedback is strongest.

The stripe soliton is always unstable to perturbations in the $y$ direction, which break it into a chain of fundamental 2D solitons. This instability takes place at a finite characteristic wavelength. When considering vortices, this instability corresponds to the possible azimuthal instabilities. For vortices of small radii the curvature can suppress these instabilities, and the vortices can be stable.

Studying the stability and dynamics of the stripe in the $x$ direction is very relevant to determine the radial dynamics 
(a)

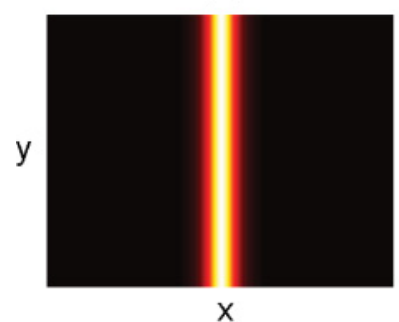

(c)

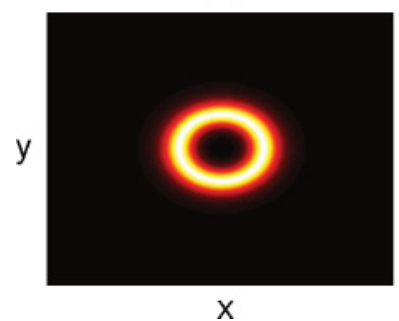

(b)

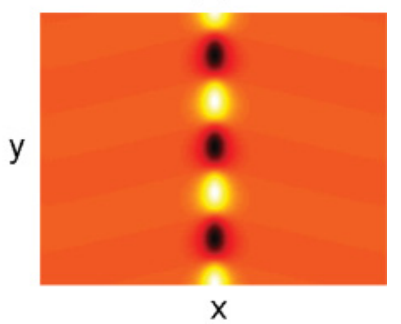

(d)

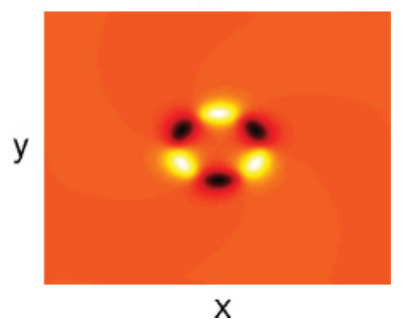

FIG. 5. (Color online) Spatial profiles of the absolute value (a) and real part (b) of the field for the unstable 2D stripe soliton (12) at $\mu=0.52$. (c) and (d): The same as (a) and (b), but for the stable vortex with $m=3$.

of large vortex rings. Moreover, the growth rate of the driftinstability in the $x$ direction is much higher than the growth rate of the instability in the $y$ direction. Then, for the stripe soliton bent into a ring with a large radius, the drift instability dominates, reducing the radius, while $y$ instability is eventually suppressed, leading to the formation of the stable vortex.

Figure 6(c) shows the largest real parts of the eigenvalues obtained from the linear stability analysis in the $x$ direction. The stripe soliton is subject to a drift instability, similar to that of the 1D soliton outlined in the previous section. In this case, the stripe starts to move in the perpendicular direction

(a)

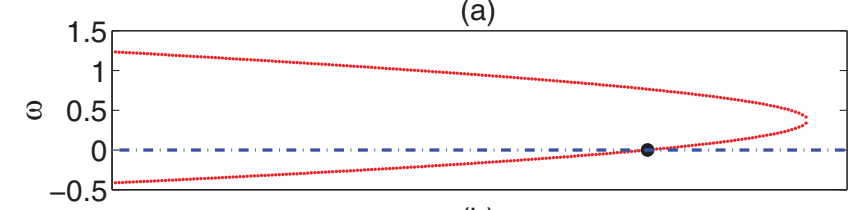

(b)

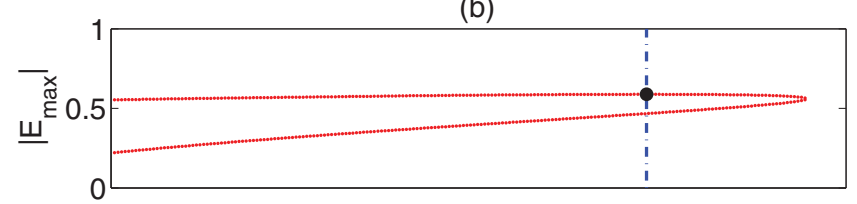

(c)

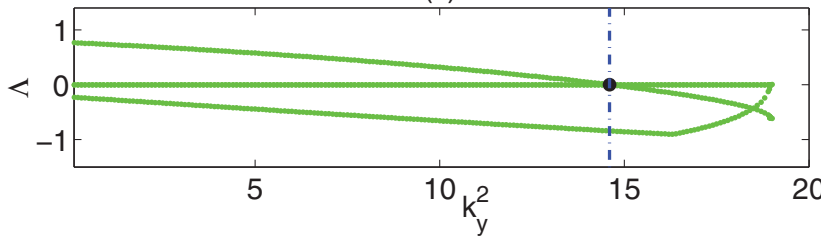

FIG. 6. (Color online) Characteristics of the continuous family of 2D stripe solitons parametrized by $k_{y}$, for $\mu=0.52$ : (a) frequency $\omega$; (b) the soliton's amplitude $\left|E_{\max }\right|$; (c) the growth rates of the unstable, neutral, and least damped eigenmodes (the stability is considered against perturbations depending only on coordinate $x$ ).

before breaking up into a chain of fundamental 2D solitons. It is noteworthy that the drift instability occurs, within numerical accuracy, at $k_{y}=k_{y}^{c}$, and hence $\omega=0$. The critical value $k_{y}^{c}$ will be very relevant below, when considering the radial dynamics of vortices.

We now proceed to the study of fully localized 2D solutions. There are two types of stable 2D modes: fundamental solitons with a bell-like intensity profile [see Fig. 2(b)] and ring-shaped vortex solitons [see Fig. 5(c)]. Vortex solutions with integer topological charge $m$ can be looked for as $E(r, \phi)=E_{0}(r) e^{i m \phi}$, where $(r, \phi)$ are polar coordinates with the origin at the pivot of the vortex. The fundamental 2D soliton corresponds to $m=0$, with the maximum at the origin. Every vortex with topological charge $m$ has a mirror-image counterpart with charge $-m$ and identical dynamical properties. Therefore, we only consider the solutions with $m \geqslant 0$.

Figure 5 highlights the similarity between the vortex and stripe solitons. Roughly speaking, the vortex may be considered as a stripe bent into a closed circle, at least for large values of $m$. Following this similarity, one can study the radial dynamics of vortices using the radial version of Eq. (2), with

$$
\Delta_{\perp}=\frac{\partial^{2}}{\partial r^{2}}+\frac{1}{r} \frac{\partial}{\partial r}-\frac{m^{2}}{r^{2}},
$$

avoiding azimuthal instabilities. As initial conditions, we use the 1D analytical solution (4), written in the radial direction:

$$
\left\{\begin{array}{l}
E(r)=E_{\max }\left\{\cosh \left[K\left(r-R_{0}\right)\right]\right\}^{-1-i \beta} e^{i \omega t}, \\
F(r)=F_{\max }\left\{\cosh \left[K\left(r-R_{0}\right)\right]\right\}^{-1-i \beta} e^{i \omega t},
\end{array}\right.
$$

where $R_{0}$ is the initial radius of the vortex. If $R_{0}$ is too small, the field decays to zero, but for $R_{0}$ large enough we observe the evolution of the position of the maximum of the field's amplitude $r_{\max }(t)$ toward the equilibrium radius $R^{\text {st }}(m)$ of the vortex soliton for each charge $m$ (see Fig. 7). If $R_{0}$ is much larger than $R^{\text {st }}(m)$, we observe shrinkage of the vortex at a constant speed, which is then followed by relaxational oscillations, as radius $R^{\text {st }}(m)$ is approached.

The constant shrinkage speed may be explained by the quasi-1D dynamics of the stripe-soliton solution. Indeed, if the initial condition has very large $r_{\max }$, the ring may be considered locally as a stripe with $k_{y} \approx 0$, which is drift-unstable (see Fig. 6). The overall curvature of the ring breaks the left-right symmetry of the stripe solution. The symmetry breaking is such that the drift naturally takes place inward (which corresponds to the action of the effective surface tension), hence the ring as a whole starts contracting at a constant speed. Thus, the first stage of the dynamics shown in Fig. 7 for $t<7$ is essentially the same as in Fig. 4. As the radius $r_{\max }$ shrinks, the effective wave number $k_{y}$ increases, eventually suppressing the drift instability and enabling the ring to relax to the stable radius $R^{\mathrm{st}}(m)$.

Running the simulations for different (large enough) values of $m$, we have produced the dependence of the equilibrium radius $R^{\text {st }}$ on topological charge $m$, which turns out to be linear (see Fig. 8). Therefore, vortex rings expand as $m$ increases, tending toward the stripe solution in the limit of $m \rightarrow \infty$. The inverse of the slope of the line in Fig. $8, R^{\text {st }}(m) / m$, is the transverse circular wave number $k_{c}$, which is, apparently, nearly constant for all vortices. The value of $k_{c}$ is, actually, 
(a)

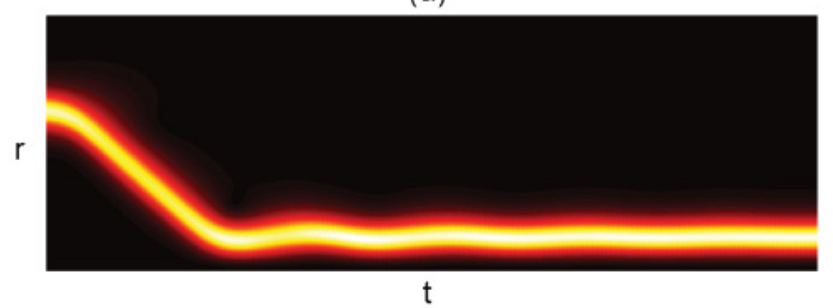

(b)

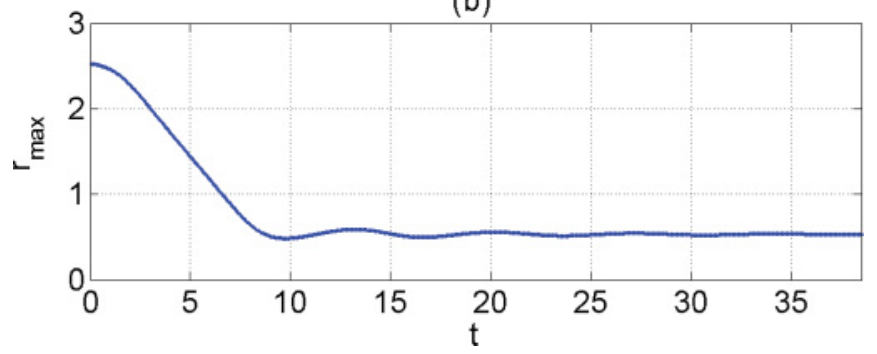

FIG. 7. (Color online) (a) The spatiotemporal dynamics of the radial profile of the field amplitude relaxing toward the equilibrium for the vortex with $m=2$ and $\mu=0.52$. (b) The evolution of position $r_{\max }$ of the maximum of the field intensity.

very close to the above-mentioned critical wave number of the drift instability of the soliton stripe, $k_{y}^{c}$.

The mechanism leading to 2D stable vortices discussed here has no counterpart in the simple curvature-driven dynamics of fronts connecting two equivalent states [30,31]. In those systems, 1D fronts in the 2D setting may be subject to modulational instabilities, but not to drifting ones. Therefore, there is no transient regime in which the ring radius evolves at a constant rate. Thus, the drift instability of the 1D soliton plays a critical role in the formation of the $2 \mathrm{D}$ vortical solitons.

The analysis presented above on the basis of the radial equation did not consider azimuthal instabilities. In fact, similar to the modulational instability of the stripe solitons, vortices with large $m$ are azimuthally unstable in the full 2D problem. Azimuthal instabilities are, however, suppressed for

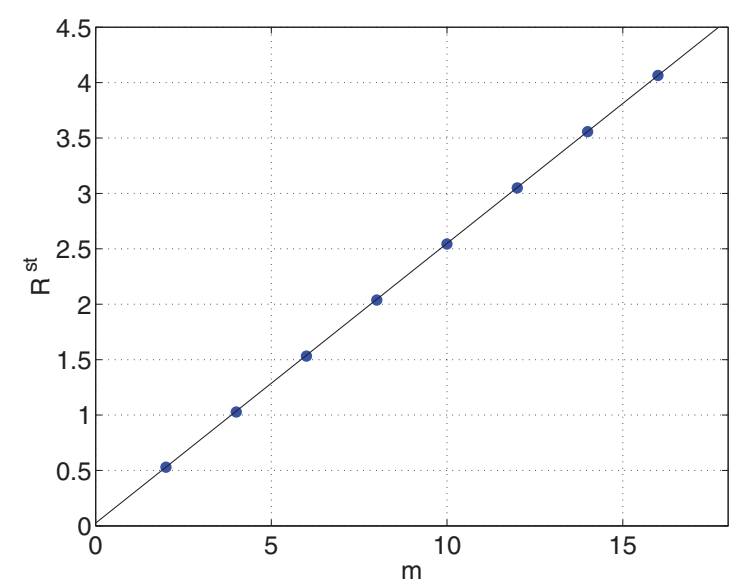

FIG. 8. (Color online) The equilibrium radius $R^{\text {st }}$ of the vortex solitons versus the topological charge $m$. Parameters are as in Fig. 7. Filled circles mark even integer values of $m$, while the solid line is a linear fitting. Figure 7 corresponds to the leftmost point in this figure.

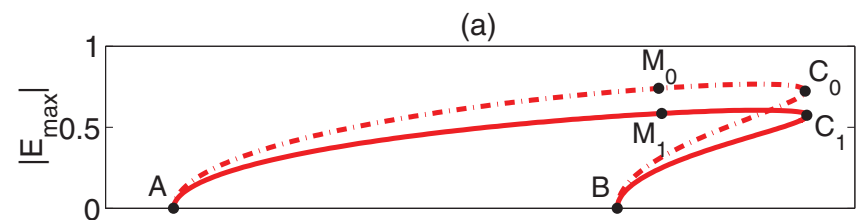

(b)

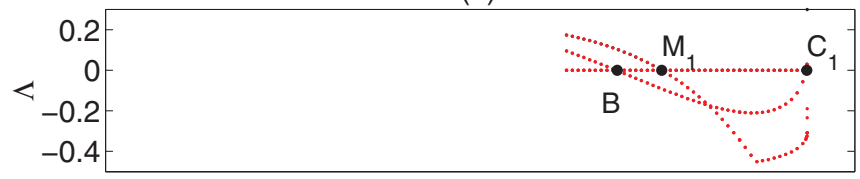

(c)

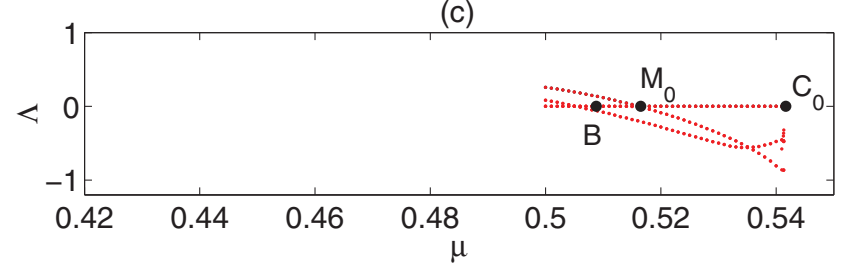

FIG. 9. (Color online) (a) The amplitude of the soliton with $m=0$ (the dashed-dotted line) and $m=1$ (the solid line) versus the pump current. (b) The growth rate of six localized eigenmodes with highest $\Lambda$, including unstable, neutral, and least damped stable ones, for the solution branch $C_{1} M_{1} A$ in (a). (c) The same as in (b), but for branch $\mathrm{C}_{0} \mathrm{M}_{0} \mathrm{~A}$ in (a). Parameters are the same as used throughout this section.

smaller topological charges, and vortices can be stable up to a certain value of $m$ [17]. Figures 5(c) and 5(d) show, for instance, a stable vortex with $m=3$. Following the method described in Ref. [17], we have generated bifurcation diagrams for the solitons with $m=0$ and 1 , and have analyzed their full stability (see Fig. 9). The fundamental soliton (vortex) is stable between points $\mathrm{M}_{0}$ and $\mathrm{C}_{0}\left(\mathrm{M}_{1}\right.$ and $\left.\mathrm{C}_{1}\right)$. Point $\mathrm{M}_{0}\left(\mathrm{M}_{1}\right)$ corresponds, as in Fig. 3, to the onset of the drift instability of the mode as a whole. The branches connecting points $\mathrm{B}$ and $\mathrm{C}_{0}\left(\mathrm{~B}\right.$ and $\mathrm{C}_{1}$ ) correspond to the solitons which play the role of the unstable separatrix. The fundamental 2D soliton and the $m=1$ vortex have quite similar bifurcation diagrams. In comparison to the diagram for 1D solitons (see Fig. 3), points $\mathrm{A}$ and $\mathrm{B}$ correspond to the limits of the region where the zero background solution is unstable against spatially uniform perturbations, hence are identical to their counterparts in Fig. 3, while points $\mathrm{C}$ and $\mathrm{M}$, corresponding to the $2 \mathrm{D}$ solitons, are located at lower values of the pump.

We have also investigated the stability of vortices with $m=2$ and 3 (not shown in the figure). Their existence region is almost identical to that of the $m=1$ vortex, while their stability region is narrower and lies inside interval $M_{1} C_{1}$, in terms of Fig. 9.

Finally, we have also observed different types of radially symmetric localized states, which feature radial oscillations in the intensity profile (see Fig. 10). These solutions have been obtained in both models, based on Eqs. (2) and (1), using the shooting method in the radial equation. Similar solutions have been observed in conservative nonlinear wave equations with the cubic nonlinearity $[32,33]$, and they can be interpreted as excited states of radial modes. The bifurcation diagrams for these excited states look qualitatively similar to that of the fundamental soliton. These solutions may be stable against 


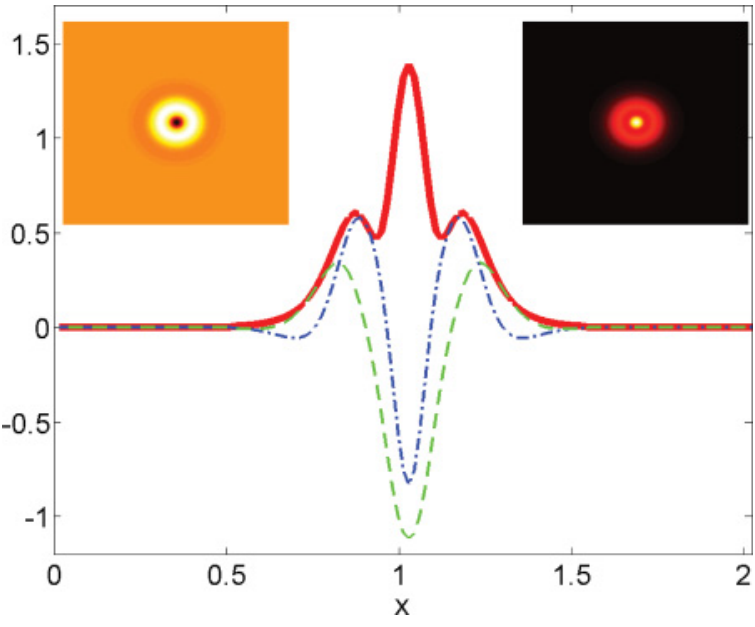

FIG. 10. (Color online) Profile of an excited radial mode. The solid line shows the absolute value of the field, while the dashed (dashed-dotted) line displays the real (imaginary) part of the field. The left-hand inset shows the $2 \mathrm{D}$ distribution of the real part [the gray (orange) background is zero, while the minimum (maximum) value corresponds to the black (white)]. The right-hand inset shows the amplitude profile (here black corresponds to zero, and white- to the maximum value of the amplitude).

radial perturbations, but they are always subject to azimuthal instability.

\section{SUMMARY}

In this paper, we have proposed a system consisting of a cubic complex Ginzburg-Landau equation coupled to an additional dissipative linear equation as a model for laser cavities with external frequency-selective feedback. We have justified the validity of the model for a range of phenomena and parameters, where the field amplitudes are not necessarily small. This has been done by showing the similarity of our results to those recently reported in the more complex models with the saturable nonlinearity [15-18]. In other contexts, this model was known before in $1 \mathrm{D}$ settings, but not in $2 \mathrm{D}$, to the best of our knowledge.

As a matter of fact, our results present an example of stable 2D solitons, both fundamental and vortical ones, in a phaseinvariant system with cubic nonlinearity. Stable fundamental 2D solitons were known before in models of other types, namely, with saturable and cubic-quintic nonlinearities (in the latter case, stable solitary vortices with $m=1$ and 2 were also known [34]), and in cubic systems with broken phase invariance $[35,36]$.

Using analytical considerations and numerical analysis, we have shown that $2 \mathrm{D}$ vortex solitons can be interpreted as stripe solitons bent into rings, which eventually suppresses the instability of the stripes and allows one to find stable vortex rings. This correspondence is clear for $m \rightarrow \infty$, but it actually holds for rather small topological charges, too, since the circular transverse wave number appears to be the same for all $m$. In such a way, we have established a connection between $1 \mathrm{D}$ and $2 \mathrm{D}$ solitons.

\section{ACKNOWLEDGMENTS}

We acknowledge financial support from MICINN (Spain) and FEDER (EU) through Grant No. FIS2007-60327 FISICOS.
[1] Y. S. Kivshar and B. A. Malomed, Rev. Mod. Phys. 61, 763 (1989).

[2] M. C. Cross and P. C. Hohenberg, Rev. Mod. Phys. 65, 851 (1993).

[3] I. S. Aranson and L. Kramer, Rev. Mod. Phys. 74, 99 (2002).

[4] C. Etrich, F. Lederer, B. A. Malomed, T. Peschel, U. Peschel, and E. Wolf, Prog. Opt. 41, 483 (2000).

[5] A. V. Buryak, P. D. Trapani, D. V. Skryabin, and S. Trillo, Phys. Rep. 370, 63 (2002).

[6] A. S. Desyatnikov, Y. S. Kivshar, L. Torner, and E. Wolf, Prog. Opt. 47, 291 (2005).

[7] Dissipative Solitons, edited by N. Akhmediev and A. Ankiewicz, Lecture Notes in Physics, Vol. 661 (Springer, New York, 2004).

[8] B. A. Malomed, Chaos 17, 037117 (2007).

[9] Y. V. Kartashov, B. A. Malomed, and L. Torner, Rev. Mod. Phys. 83, 247 (2011).

[10] B. S. Kerner and V. V. Osipov, in Autosolutions: A New Approach to Problems of Self-Organization and Turbulence, Fundamental Theories of Physics, Vol. 61 (Kluwer Academic, Dordrecht, 1994).

[11] H.-G. Purwins, H. U. Bödeker, and Sh. Amiranashvili, Adv. Phys. 59, 485 (2010).
[12] T. Elsass, K. Gauthron, G. Beaudoin, I. Sagnes, R. Kuszelewicz, and S. Barbay, Eur. Phys. J. D 59, 91 (2010).

[13] L. A. Lugiato and R. Lefever, Phys. Rev. Lett. 58, 2209 (1987).

[14] P. Genevet, S. Barland, M. Giudici, and J. R. Tredicce, Phys. Rev. Lett. 104, 223902 (2010).

[15] P. V. Paulau, D. Gomila, T. Ackemann, N. A. Loiko, and W. J. Firth, Phys. Rev. E 78, 016212 (2008).

[16] P. V. Paulau, D. Gomila, P. Colet, M. A. Matias, N. A. Loiko, and W. J. Firth, Phys. Rev. A 80, 023808 (2009).

[17] P. V. Paulau, D. Gomila, P. Colet, N. A. Loiko, N. N. Rosanov, T. Ackemann, and W. J. Firth, Opt. Express 18, 8859 (2010).

[18] P. V. Paulau, A. J. Scroggie, A. Naumenko, T. Ackemann, N. A. Loiko, and W. J. Firth, Phys. Rev. E 75, 056208 (2007).

[19] N. Radwell, C. McIntyre, A. Scroggie, G.-L. Oppo, W. J. Firth, and T. Ackemann, Eur. Phys. J. D 59, 121 (2010).

[20] T. Ackemann, W. J. Firth, and G.-L. Oppo, Adv. At. Mol. Opt. Phys. 57, 323 (2009).

[21] M. Yousefi and D. Lenstra, IEEE J. Quantum Electron. 35, 970 (1999).

[22] D. Mihalache, D. Mazilu, F. Lederer, H. Leblond, and B. A. Malomed, Phys. Rev. A 77, 033817 (2008).

[23] H. Leblond, B. A. Malomed, and D. Mihalache, Phys. Rev. A 80, 033835 (2009). 
[24] D. Mihalache, D. Mazilu, V. Skarka, B. A. Malomed, H. Leblond, N. B. Aleksić, and F. Lederer, Phys. Rev. A 82, 023813 (2010).

[25] V. Skarka, N. B. Aleksić, H. Leblond, B. A. Malomed, and D. Mihalache, Phys. Rev. Lett. 105, 213901 (2010).

[26] J. Atai and B. A. Malomed, Phys. Lett. A 246, 412 (1998).

[27] Here we have presented the results for $\omega_{m}=-270$, for which the marginal stability curve is the same as in Fig. 1, but displaced by 20 units to the left-hand side. For $\omega_{m} \approx-250$, moving solitons instead of quiescent ones are observed in the final state.

[28] B. A. Malomed and H. G. Winful, Phys. Rev. E 53, 5365 (1996).

[29] J. Atai and B. A. Malomed, Phys. Rev. E 54, 4371 (1996).
[30] P. L. Christiansen, N. Grø nbech-Jensen, P. S. Lomdahl, and B. A. Malomed, Phys. Scr. 55, 131 (1997).

[31] D. Gomila, P. Colet, G.-L. Oppo, and M. San Miguel, Phys. Rev. Lett. 87, 194101 (2001).

[32] Z. K. Yankauskas, Izv. Vyssh. Uchebn. Zav. Radiofiz. 9, 412 (1966) [Sov. Radiophys. 9, 261 (1966)].

[33] J. M. Soto-Crespo, D. R. Heatley, E. M. Wright, and N. N. Akhmediev, Phys. Rev. A 44, 636 (1991).

[34] L. C. Crasovan, B. A. Malomed, and D. Mihalache, Phys. Rev. E 63, 016605 (2000).

[35] L. S. Tsimring and I. S. Aranson, Phys. Rev. Lett. 79, 213 (1997).

[36] I. V. Barashenkov, N. V. Alexeeva, and E. V. Zemlyanaya, Phys. Rev. Lett. 89, 104101 (2002). 